\title{
Correspondence
}

\section{Airway anesthesia: the toothpaste method}

To the Editor:

I enjoyed the description by Chung et al. of a simple technique for achieving topical anesthesia of the airway. ${ }^{1}$ I have, for many years, used a variation of the technique that Chung et al. have examined in detail and identified it as the "toothpaste method". Rather than the liquid lidocaine preparation which Chung et al. instill on the base of the tongue, I employ lidocaine 5\% ointment. This has the advantage that it does not require transfer to an intermediary administration device.

As early as possible, I ask that the patient stick out his/her tongue as far as possible. I explain that I am going to put "a line of toothpaste down the middle of your tongue". I then instruct the patient to oppose the tongue to the roof of the mouth and hold it there as long as possible while the lidocaine ointment "melts". I do this with the patient supine. During the time taken to achieve intravenous access, transport the patient to the operating room and apply monitors, I usually administer a second line of toothpaste.

Five or more minutes after the initial administration, the patient may begin to cough, and is why I begin to apply the second line of toothpaste. I now believe that the cough represents the ointment reaching the carina or lower portions of the airway rather than being an indication of incomplete anesthesia of the glottis.

I discourage trainees in anesthesia from using more invasive injection techniques and have resisted suggestions to supplement the toothpaste technique with those same blocks.

John C. Drummond MD FRCPC

San Diego, California, USA

Reference

1 Chung DC, Mainland P-A, Kong AS. Anesthesia of the airway by aspiration of lidocaine. Can J Anesth 1999; 46: 215-9.

REPLY:

Thank you for the opportunity to comment on "Airway anesthesia: the toothpaste method". ${ }^{1}$ To anesthetize the airway below the glottis in our aspiration technique, ${ }^{2}$ we used tongue traction to discourage swallowing and to promote aspiration of the lidocaine solution. It is unclear why the patient does not simply swallow the lidocaine ointment in the toothpaste method described by Dr. Drummond. Although he has observed anesthesia at and below the glottis, there remains doubt of the quality of anesthesia. It deserves a formal and comparative study.

\section{D.C. Chung MD FRCA FRCPC FHKCA FHKAM}

Shatin, Hong Kong, China

\section{References}

1 Drummond JC. Airway anesthesia: the toothpaste method. Can J Anesth 2000; 47: 94.

2 Chung DC, Mainland P-A, Kong AS. Anesthesia of the airway by aspiration of lidocaine. Can J Anesth 1999; 46: 215-9.

\section{Models for difficult laryngoscopy}

To the Editor:

Naguib et al., further elucidated the relationship of airway measures with the risk of difficult intubation. ${ }^{1}$ Their clinical model, however, may not be applicable for use in clinical practice.

First, "all known" clinical risk criteria were not included in the analysis. For example, atlanto-occipital extension was not included as a predictor measure only head and neck movement was included as part of the Wilson risk sum score, which is unreliable. Other important measures may have altered their model, such as that described by Bellhouse et al. ${ }^{2}$ All potentially important variables should be assessed to ensure that the prediction model is reliable and valid.

Second, their models were developed on only 25 patients who were difficult to intubate. Up to ten clinical measures were included in their multivariable analysis: for every predictor variable, there were only 2.5 patients with difficult intubation. However, to ensure that multivariable models do not suffer from over-fitting, where they perform well only on the sample from which they were derived, the outcome: variable ratio should be greater than $5: 1 .^{3}$

Therefore, the model is most likely over-fitted and may not be applicable to other populations. There are at least two methods of validation to determine if the 